Erschienen in: Fridays for Future - Die Jugend gegen den Klimawandel : Konturen der weltweiten Protestbewegung / Haunss, Sebastian; Sommer, Moritz (Hrsg.). - Bielefeld: transcript, 2020. - S. 205-226. - ISBN 978-3-8376-5347-2

http://dx.doi.org/10.14361/9783839453476-010

\title{
10. Die gesellschaftliche Unterstützung von Fridays for Future
}

Sebastian Koos und Franziska Lauth

Innerhalb weniger Wochen und Monate haben Fridays for Future eine umfassende gesellschaftliche und politische Debatte über die Klimakrise und die deutsche Klimapolitik angestoßen. Dabei haben nicht nur die Form des Protests, der Schul- oder Klimastreik, sondern auch das Ausmaß der Klimabedrohung und die Forderung nach nötigen gesellschaftlichen und klimapolitischen Veränderungen kontroverse Diskussionen ausgelöst. Während sich viele Bürger_innen, Politiker_innen und Prominente hinter Fridays for Future stellten und zahlreiche andere Gruppen, wie Parents for Future oder Scientists for Future, entstanden, regte sich auch breiter Widerstand sowohl gegen die Proteste als auch gegen die politischen Forderungen. In diesem Kapitel beschäftigen wir uns mit der gesellschaftlichen Unterstützung der Fridays for Future-Bewegung. Darunter verstehen wir ganz allgemein, dass verschiedene Gruppen der Bevölkerung die Ziele und Protestformen einer Bewegung positiv bewerten und diesen mindestens aufgeschlossen gegenüberstehen. Die Unterstützung kann aber auch weiter reichen und sich in der Bereitstellung von Ressourcen oder der aktiven Teilnahme an Protesten äußern.

Öffentliche Meinung und Unterstützung sind aus mehreren Gründen von zentraler Bedeutung für soziale Bewegungen. Erstens bestimmt die öffentliche Meinung die Legitimität von sozialen Bewegungen im Sinne ihrer Anerkennung oder Akzeptanz. Legitimität wiederum ist eine mögliche Voraussetzung, um neue Anhänger_innen und Teilnehmer_innen zu rekrutieren und andere relevante Ressourcen, wie beispielsweise finanzielle Unterstützung, zu mobilisieren. Zweitens spielen Einstellungen und Meinungen eine wichtige Rolle im politischen Entscheidungsprozess. Die öffentliche Meinung kann Reformen blockieren, wenn Politiker_innen befürchten, un- 
populäre Reformvorschläge könnten sie Wähler_innenstimmen kosten. Neben der direkten Auseinandersetzung mit politischen Akteuren üben soziale Bewegungen daher auch über die öffentliche Meinung politischen Einfluss aus. Abschließend gibt die gesellschaftliche Unterstützung auch darüber Aufschluss, wie groß die potenzielle Bereitschaft ist, privates Verhalten wie zum Beispiel Konsummuster auch unabhängig von politischen Vorgaben zu verändern.

Soziale Bewegungen können unterschiedliche Arten der Unterstützung erfahren, die jeweils ein unterschiedliches Ausmaß an Engagement und Einsatz voraussetzen (McCarthy/Zald 1977). Wir unterscheiden hierbei zwischen einer allgemeinen positiven Meinung oder Einstellung zu Fridays for Future (Unterstützer_innen), der potenziellen Bereitschaft, an Protesten teilzunehmen (Anhänger_innen), sowie der wirklichen Teilnahme an Protesten (Teilnehmer_innen) (siehe auch Ennis/Schreuer 1987). Die verschiedenen Unterstützungsformen können wie konzentrische Kreise verstanden werden, die mit zunehmendem Engagement eine immer kleinere Personenzahl beinhalten. Die Unterstützung für eine Bewegung und die Bereitschaft, an Protesten teilzunehmen, sind Voraussetzungen für eine Teilnahme. Aber nicht alle, die prinzipiell bereit sind, an Protesten teilzunehmen, tun dies auch wirklich, beispielsweise aus Mangel an Zeit, aufgrund räumlicher Distanz oder fehlenden Kontakten zu anderen Protestteilnehmer_innen (Klandermans/Oegema 1987).

Aber welche Unterstützung hat Fridays for Future in der Bevölkerung eigentlich? Wer ist prinzipiell bereit, an Demonstrationen teilzunehmen, und wer nimmt an diesen auch tatsächlich teil? Und inwieweit sind Wähler_innen bereit, Forderungen nach klimapolitischen Maßnahmen, etwa in Form einer $\mathrm{CO} 2$-Steuer, zu unterstützen oder ihren Lebensstil zu ändern? Diese drei Fragen werden wir im folgenden Kapitel beantworten. Dabei greifen wir auf eine repräsentative Bevölkerungsbefragung zurück, die im Mai 2019 unter fast 5.000 Personen im Rahmen des German Internet Panel (GIP) durchgeführt wurde. ${ }^{1}$ Die Befragungswelle enthielt ein Ad-hoc-Modul,

1 Das Cerman Internet Panel (GIP) ist eine Längsschnittstudie, die seit 2014 am Sonderforschungsbereich »Politische Ökonomie der Reformen« (SFB 884) an der Universität Mannheim durchgeführt wird. Um die Repräsentativität der Stichprobe nach Alter, Geschlecht, Bildung und Wohnort zu verbessern, verwenden wir für die Analyse Gewichte. Das Mindestalter, um an der Studie teilzunehmen, beträgt 16 Jahre. 
welches sich mit den Fridays for Future-Protesten beschäftigte (Koos/Naumann 2019). Das GIP basiert auf einer Zufallsstichprobe der Bevölkerung in Deutschland im Alter von 16 bis 75 Jahren. Auf Basis dieser Daten werden wir im Folgenden die Einstellungen der Bevölkerung zu den Klimastreiks, die geplante und selbstberichtete Teilnahme an diesen Protesten, sowie die Unterstützung der von Fridays for Future vorgeschlagenen $\mathrm{CO}_{2}$-Steuer und Veränderungen des privaten Lebensstils auswerten. Dabei werden wir insbesondere auf soziale Unterschiede im Hinblick auf Geschlecht, Alter, Bildungsstand und politische Orientierung eingehen. Im Hinblick auf die Protestteilnahme vergleichen wir die Befunde der GIP-Daten mit Ergebnissen einer Protestbefragung des Instituts für Protest- und Bewegungsforschung (Sommer u. a. 2019; Sommer u.a., Kapitel 2).

Abbildung 10.1: Die konzentrischen Kreise der gesellschaftlichen Unterstützung von Fridays for Future

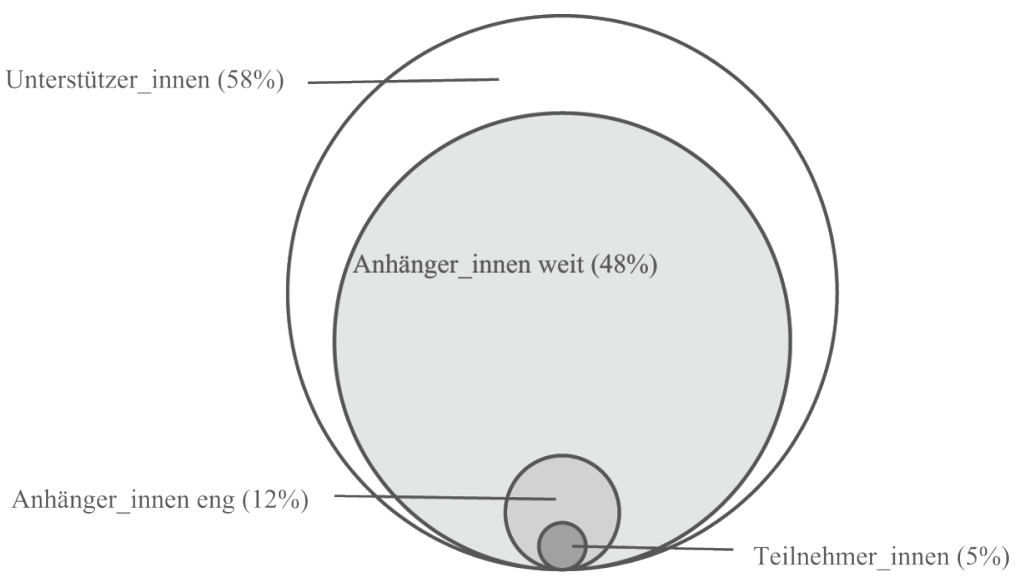

Unsere Analysen zeigen einen großen Rückhalt von Fridays for Future in der Gesellschaft, der sich allerdings stark zwischen gesellschaftlichen Gruppen unterscheidet. In Abbildung 10.1 haben wir die konzentrischen Kreise der Unterstützung dargestellt. Mehr als die Hälfte der Bevölkerung steht den Klimastreiks positiv gegenüber (Unterstützer_innen). Etwas mehr als vier von zehn Befragten geben an, prinzipiell bereit zu sein, zukünftig an einer Klimademonstration teilzunehmen (Anhänger_innen weit), wobei nur eine von zehn Personen »auf jeden Fall« teilnehmen will (Anhänger_innen eng). 
Allerdings hatten nur etwa fünf von 100 Befragten zum Zeitpunkt der Befragung im Mai 2019 wirklich teilgenommen (Teilnehmer_innen). Von diesen konzentrischen Kreisen lassen sich zwei weitere Gruppen unterscheiden, die Fridays for Future entweder neutral gegenüberstehen (21 Prozent) oder die Bewegung ablehnen (ebenfalls 21 Prozent). Die Meinung zu klimapolitischen Maßnahmen ist insgesamt sehr polarisiert, ebenso gibt es starke Unterschiede im selbstberichteten Wandel hin zu klimafreundlichen Lebensstilen. Über alle Dimensionen hinweg finden wir große Unterschiede im Hinblick auf Alter, Geschlecht, Bildungsstand und politische Orientierung. Im Folgenden diskutieren wir kurz bestehende Forschung zur Legitimität sozialer Bewegungen sowie Einstellungen zu Umwelt- und Klimaschutz und zeigen, wie diese sozial strukturiert sind. Danach präsentieren wir die Ergebnisse unserer Analysen zu den einzelnen Dimensionen der Unterstützung von Fridays for Future. Anschließend zeigen wir, welchen Einfluss die Einstellungen zu Fridays for Future auf die Unterstützung einer $\mathrm{CO}_{2}$-Steuer und auf Veränderungen des Lebensstils haben. Eine kurze Zusammenfassung und Diskussion beschließen den Beitrag.

\section{Die soziale Basis der Klimaschutzbewegung im Spiegel bestehender Forschung}

Die meisten Studien, die sich mit der gesellschaftlichen Unterstützung von sozialen Bewegungen beschäftigen, untersuchen in erster Linie die öffentliche Meinung hinsichtlich der Themen oder Probleme, die eine soziale Bewegung anspricht. So gibt es zahlreiche Studien, die Einstellungen zum Klimawandel oder allgemeiner zu Umweltschutz untersuchen (Van Liere/ Dunlap 1980; Jones/Dunlap 1992; Dietz/Stern/Guagnano 1998; Dietz/Kalof/ Stern 2002; Franzen/Meyer 2010; McCright/Dunlap/Marquart-Pyatt 2016). Selten hingegen werden bislang die Einstellungen $\mathrm{zu}$ spezifischen sozialen Bewegungen und ihren Protestformen in den Blick genommen (Andrews/ Beyerlein/Farnum 2015). Die Frage danach, warum Teile der Gesellschaft trotz etablierter Möglichkeiten politischer Einflussnahme, wie beispielsweise Wählen oder Petitionen unterschreiben, Proteste gutheißen, ist bislang unzureichend beantwortet. Aber sie ist wichtig, da die öffentliche Einstellung gegenüber einer sozialen Bewegung den Aktivist_innen das Ausmaß potenzieller Unterstützung und die mögliche Zahl zukünftiger Protestteil- 
nehmender erst sichtbar macht (Klandermans 1984). Die gesellschaftliche Unterstützung und damit die Legitimität einer sozialen Bewegung ist letztlich entscheidend dafür, Mobilisierungsziele wie etwa den Aufbau finanzieller und organisationaler Ressourcen zu erreichen und schließlich für die Möglichkeit politischer Einflussnahme (Muñoz/Anduiza 2019).

In einer einschlägigen empirischen Studie untersucht Rohrschneider (1990), welche gesellschaftlichen Strukturen und welche individuellen und psychologischen Faktoren der öffentlichen Unterstützung von Umweltbewegungen zuträglich sind. Auf Basis einer Umfrage in vier europäischen Ländern zeigt er, dass insbesondere postmaterialistische Wertevorstellungen (wie die Betonung von Umweltschutz und Selbstentfaltung), linke politische Einstellungen sowie individuelle Problemwahrnehmung die gesellschaftliche Unterstützung der Umweltbewegung erklären können (Rohrschneider 1990). In einer klassischen Studie der Bürgerrechtsbewegung in den USA zeigt Olsen (2014), dass die wahrgenommene Legitimität der Protestaktivitäten in der Bevölkerung stark vom Bildungsgrad, dem Alter und individuellen politischen Präferenzen abhängt. Andrews und Kollegen (2015) zeigen ebenfalls anhand der Bürgerrechtsbewegung in den USA, dass Menschen, die häufiger mit Protesten in Kontakt kommen, diesen gegenüber positiver eingestellt sind. Darüber hinaus stellen sie fest, dass höhere Schulbildung die Toleranz gegenüber Protesten erhöht. Andere Studien untersuchen die Zustimmung zu Protesten in Abhängigkeit der Protestform und zeigen, dass insbesondere gewalttätiger Protest die gesellschaftliche Unterstützung reduzieren kann (Thomas/Louis 2013; Muñoz/Anduiza 2019). Stern und Kollegen (1999) zeigen, dass die gesellschaftliche Unterstützung einer sozialen Bewegung davon abhängt, ob Beobachter_innen die Werte und Ziele einer Bewegung akzeptieren. Vor diesem Hintergrund liefern Studien, die sich mit Einstellungen zur Umwelt und dem Klimawandel auseinandersetzen, mögliche Erkenntnisse zur gesellschaftlichen Unterstützung von Fridays for Future.

Studien zum Umweltbewusstsein zeigen, dass Frauen größere Besorgnis über Umweltprobleme äußern als Männer (Blocker/Eckberg 1997; Dietz/ Kalof/Stern 2002). Gleichzeitig sind jüngere Menschen besorgter über Umweltprobleme als ältere Menschen oder ältere Generationen (Van Liere/Dunlap 1980; Jones/Dunlap 1992; Dietz/Stern/Guagnano 1998; Franzen/Meyer 2010). Eine mögliche Erklärung hierfür ist laut Van Liere und Dunlap (1980), dass jüngere Menschen weniger stark in das heimische Wirtschaftssystem 
und allgemein in die Gesellschaftsordnung integriert sind und daher Lösungen für existierende Umweltprobleme auch weniger als Bedrohung für diese soziale Ordnung und die Wirtschaft ansehen. Im Hinblick auf den Bildungsgrad zeigen zahlreiche Studien, dass Befragte mit höherer Bildung im Schnitt größere Besorgnis in Bezug auf Umweltprobleme äußern (Van Liere/ Dunlap 1980; Dietz/Stern/Guagnano 1998; Tjernström/Tietenberg 2008). Bildung kann als kognitive Ressource betrachtet werden, die das Verständnis komplexer Probleme - wie das des Klimawandels - erhöht und die Wissen und Erfahrung bereitstellt, wodurch politische Beteiligung ermöglicht wird (Kvaløy/Finseraas/Listhaug 2012).

Bezüglich der politischen Einstellung wird angenommen, dass politisch links orientierte Menschen eher über die Umwelt besorgt sind als Menschen, die sich auf der rechten Seite des parteipolitischen Spektrums verorten (Neumayer 2004). Kvaløy und andere (2012) finden heraus, dass moderat rechts eingestellte Menschen weniger stark über die globale Erwärmung besorgt sind als Menschen in der politischen Mitte. Moderat links eingestellte Bürger_innen zeigen allerdings keinen signifikanten Unterschied in ihrer Besorgnis im Vergleich zu Bürger_innen der politischen Mitte. Lediglich bei extrem links eingestellten Menschen ist ein Unterschied festzustellen (Kvaløy/Finseraas/Listhaug 2012). Auch McCright, Dunlap und MarquartPyatt (2016) finden einen Unterschied hinsichtlich der Parteienideologie für westeuropäische Länder: Links eingestellte Bürger_innen glauben mit höherer Wahrscheinlichkeit an den anthropogenen Klimawandel und nehmen diesen eher als ernstes Problem wahr als rechts eingestellte Bürger_innen. Sie zeigen entsprechend auch eine höhere Bereitschaft, Kosten für Klimaschutzmaßnahmen in Kauf zu nehmen und Politiken zu unterstützen, die die Treibhausgasemissionen verringern (McCright/Dunlap/Marquart-Pyatt 2016).

Im nächsten Abschnitt untersuchen wir, ob sich ähnliche soziale Gradienten für die junge Klimabewegung finden lassen.

\section{Einstellungen zu Fridays for Future}

Die Einstellungen zu Fridays for Future wurden im GIP folgendermaßen abgefragt: »Seit mehreren Monaten demonstrieren in Deutschland freitags Schülerinnen und Schüler unter dem Motto >Fridays for Future während der 
Schulzeit für mehr Klimaschutz. Manche stehen diesen Demonstrationen kritisch gegenüber, andere finden es gut, dass die Jugendlichen protestieren. Haben Sie Verständnis für die Freitagsdemonstrationen der Schülerinnen und Schüler gegen den Klimawandel?« Abbildung 10.2 zeigt die allgemeine Unterstützung für Fridays for Future nach Geschlecht und Altersgruppe.

Abbildung 10.2: Verständnis für Fridays for Future nach Alter und Geschlecht

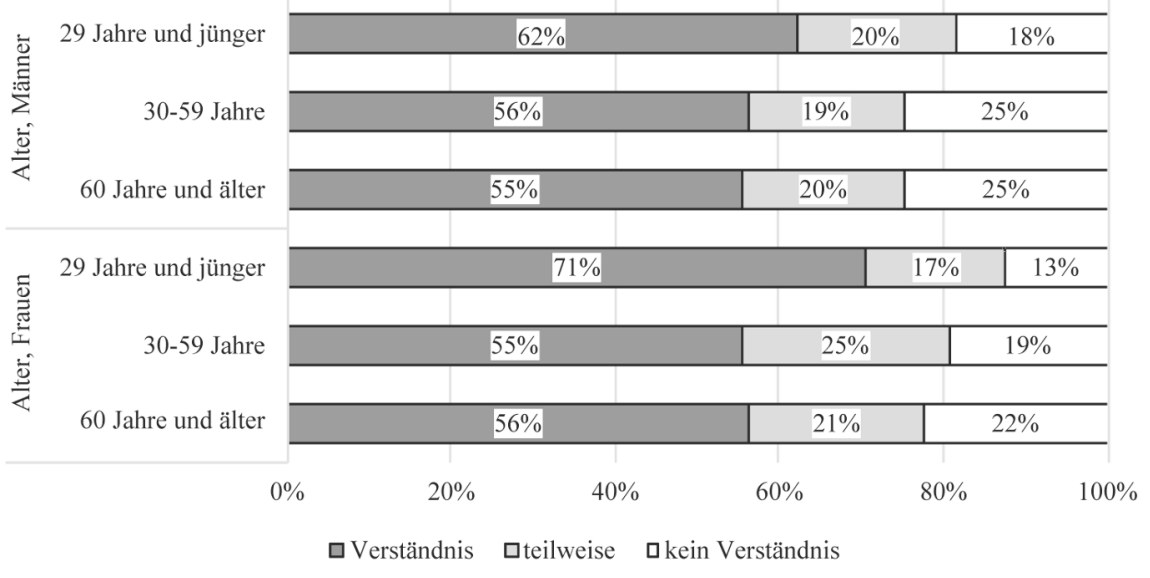

Hinweis: Antwortkategorien: Ja, voll und ganz; eher ja; teilweise; eher nein; Nein, ich habe kein Verständnis dafür. Zustimmung und Ablehnung wurden jeweils zusammengefasst.

Wenig überraschend zeigen unsere Daten, dass die Unterstützung insbesondere unter jungen Befragten sehr hoch ist, wobei auch die Unterstützung über alle Altersgruppen hinweg über 50 Prozent liegt. Lediglich in der jüngsten Gruppe der Befragten äußern Frauen mehr Verständnis für Fridays for Future als gleichalte Männer. Dies bestätigt die Befunde bestehender Forschung. Im Hinblick auf die Bildungsabschlüsse finden wir den erwarteten Einfluss höherer Bildungsabschlüsse. Mehr als zwei Drittel derer, die einen höheren Bildungsabschluss besitzen, zeigen Verständnis für die Klimabewegung. Die höchste Unterstützung erfährt die Klimabewegung jedoch von den Befragten, die sich noch in schulischer Ausbildung befinden (Abb. 10.3). 
Abbildung 10.3: Verständnis für Fridays for Future-Proteste nach Bildungsstand

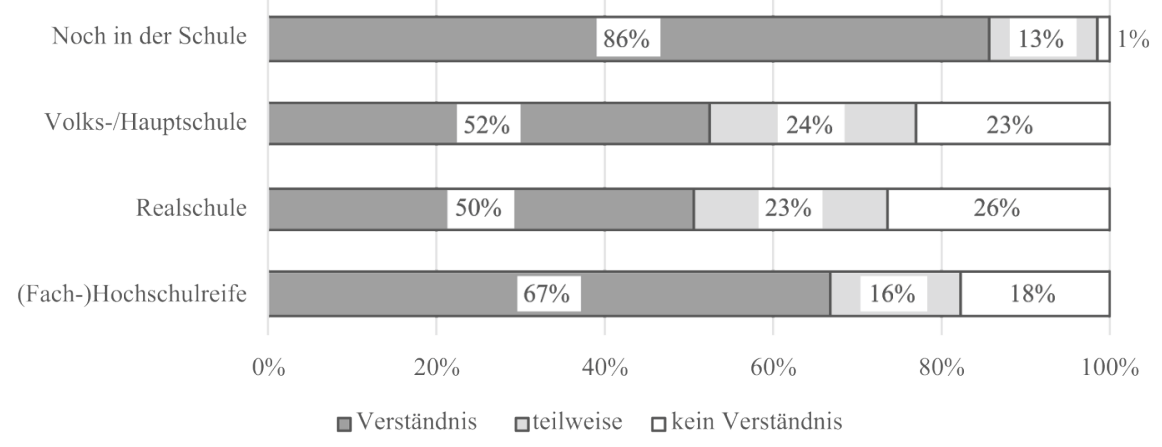

Im Hinblick auf die politische Identifikation lässt sich erkennen, dass die Unterstützung für Fridays for Future bei Personen, die noch nicht wahlberechtigt sind, am höchsten ist. In Bezug auf die Identifikation mit etablierten Parteien zeigt sich der angenommene Links-rechts-Gradient. So finden sich unter den Anhänger_innen der Grünen, der Linken und der SPD die meisten Unterstützer_innen der Klimabewegung. Von den Wähler_innen der CDU/CSU und FDP steht jeweils knapp die Hälfte Fridays for Future positiv gegenüber. Selbst bei Menschen, die sich mit der AfD identifizieren, finden sich noch Unterstützer_innen für die Klimaproteste, mit einem Anteil von einem Viertel jedoch deutlich weniger (Abb. 10.4).

\section{Potenzielle Teilnahmebereitschaft und Teilnahme an Klimademonstrationen}

Eine positive öffentliche Meinung ist eine erste wichtige Dimension im Verständnis der gesellschaftlichen Unterstützung von Fridays for Future. Dies ist allerdings nur eine erste Bedingung für die Mobilisierung von Teilnehmer_innen und Wähler_innen (van Stekelenburg/Klandermans 2013). Im zweiten Schritt untersuchen wir nun die potenzielle Teilnahmebereitschaft an Fridays for Future-Demonstrationen. In der GIP-Umfrage wurden die Teilnehmer_innen gefragt, ob "Sie in nächster Zeit an einer solchen KlimaDemonstration teilnehmen (würden), wenn diese in der Nähe Ihres Wohnortes stattfindet«? Die Befragten konnten antworten: »Ich würde auf jeden 
Abbildung 10.4: Verständnis für Fridays for Future-Proteste nach Parteiideologie

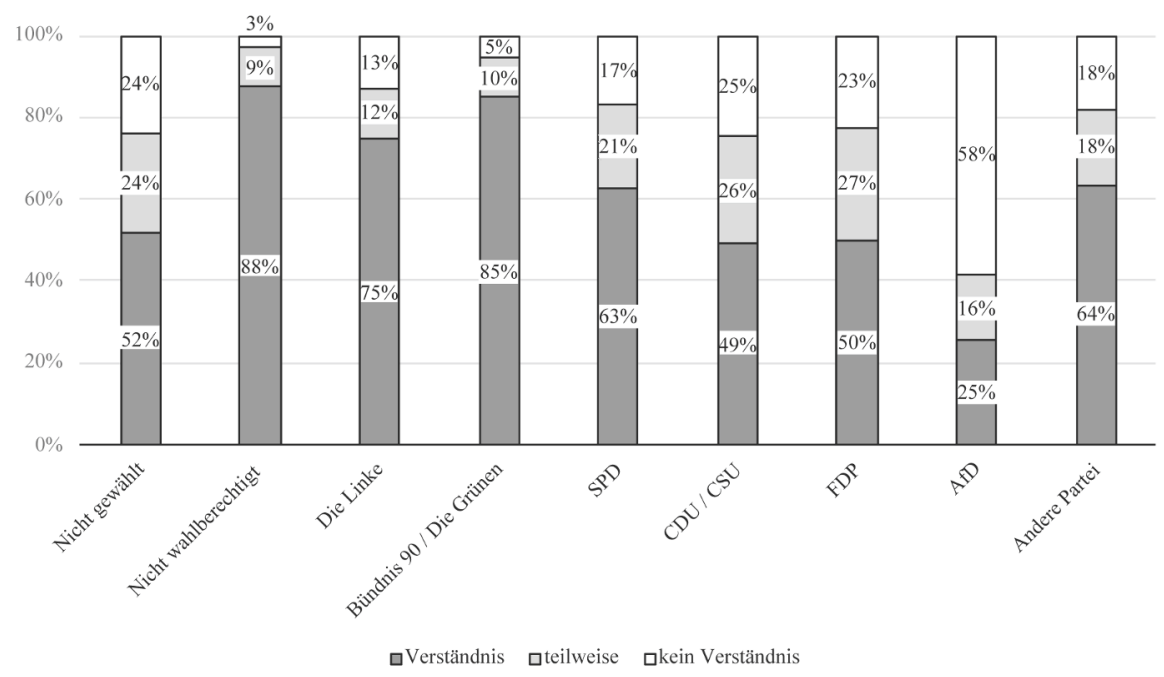

Fall an einer Klima-Demonstration teilnehmen; Ich würde wahrscheinlich an einer Klima-Demonstration teilnehmen; Ich würde nicht an einer Klima-Demonstration teilnehmen.« In Abbildung 10.5 ist die prospektive Teilnahme nach Geschlecht und Alter getrennt dargestellt. Die Antwortmuster ähneln den Einstellungsmustern von oben. Jedoch sehen wir, dass die prospektive Bereitschaft bei jungen Frauen doppelt so hoch ist wie bei gleichaltrigen Männern. Deutlich zu erkennen ist, dass Teilnahmebereitschaft an zukünftigen Protesten eine größere Hürde darstellt, als »bloßes« Verständnis gegenüber der Bewegung aufzubringen. Über alle Altersgruppen hinweg und geschlechterunabhängig zeigt die Mehrheit der Befragten eine positive Einstellung gegenüber Fridays for Future, wohingegen nur eine Minderheit uneingeschränkt bereit ist, tatsächlich (auf jeden Fall) aktiv teilzunehmen. Immerhin etwas mehr als ein Drittel aller Altersgruppen kann sich vorstellen, wahrscheinlich teilzunehmen. Jedoch schließt knapp mehr als die Hälfte aller Gruppen - mit Ausnahme junger Frauen - eine Protestteilnahme gänzlich aus. 
Abbildung 10.5: Potenzielle Teilnahmebereitschaft an Fridays for FutureProtesten nach Alter und Geschlecht

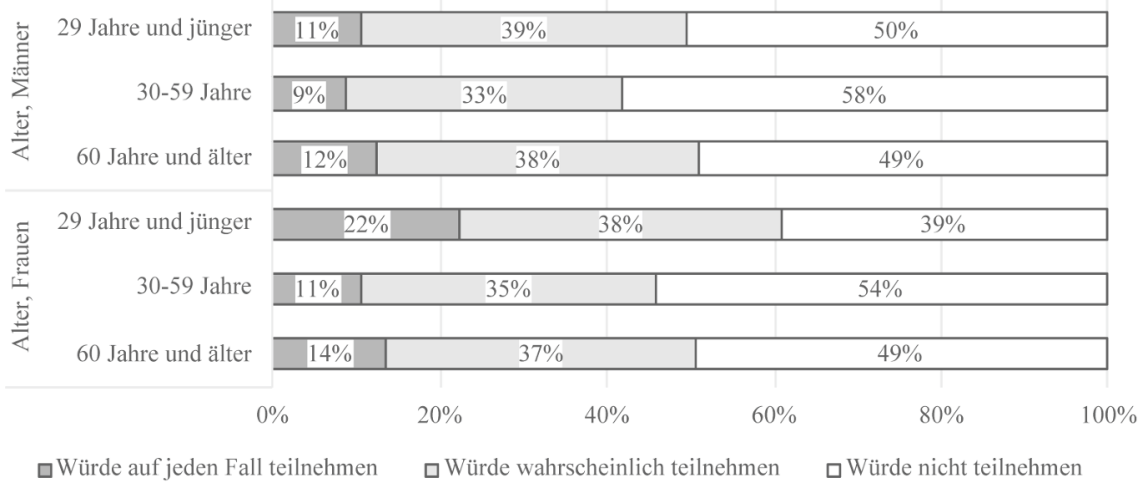

Beim Bildungsstand zeigt sich ebenfalls ein vergleichbares Bild (Abb. 10.6). Potenzielle Teilnehmer_innen sind vor allem Schüler_innen. Menschen mit Realschulabschluss zeigen insgesamt die geringste Bereitschaft, an den Protesten teilzunehmen. Die Parteiidentifikation stellen wir hier nicht explizit dar. Die Teilnahmebereitschaft ist insbesondere bei Nichtwahlberechtigten sowie bei Wähler_innen der Grünen und der Linken mit jeweils über 20 Prozent vergleichsweise hoch. Unter Wähler_innen von CDU/CSU und FDP hingegen ist sie mit jeweils etwa fünf Prozent relativ gering.

Abbildung 10.6: Potenzielle Teilnahmebereitschaft an Fridays for FutureProtesten nach Bildungsstand

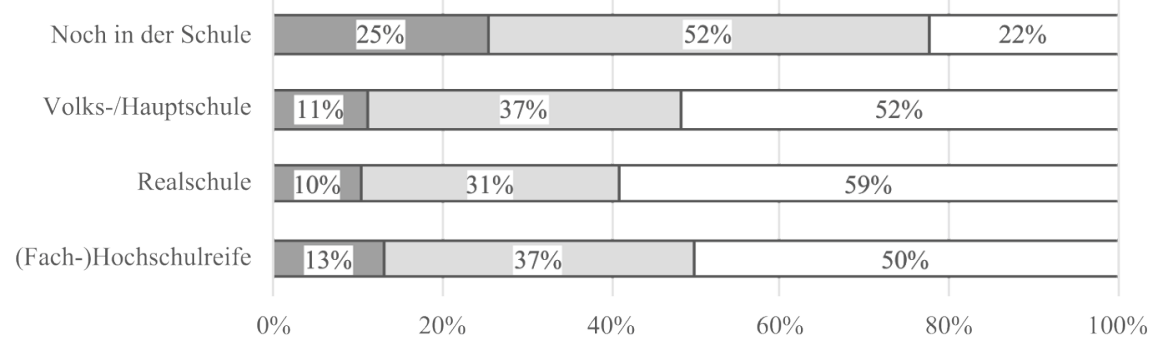


Betrachten wir nun den kleinsten konzentrischen Kreis, die aktiven Teilnehmer_innen der Klimaproteste, deren Engagement für die Bewegung am stärksten ist. Die Studienteilnehmer_innen wurden im GIP nach ihrer Teilnahme an einer Klimademonstration in den letzten sechs Monaten gefragt (Abb. 10.7).

Abbildung 10.7: Protestteilnahme nach Alter und Geschlecht

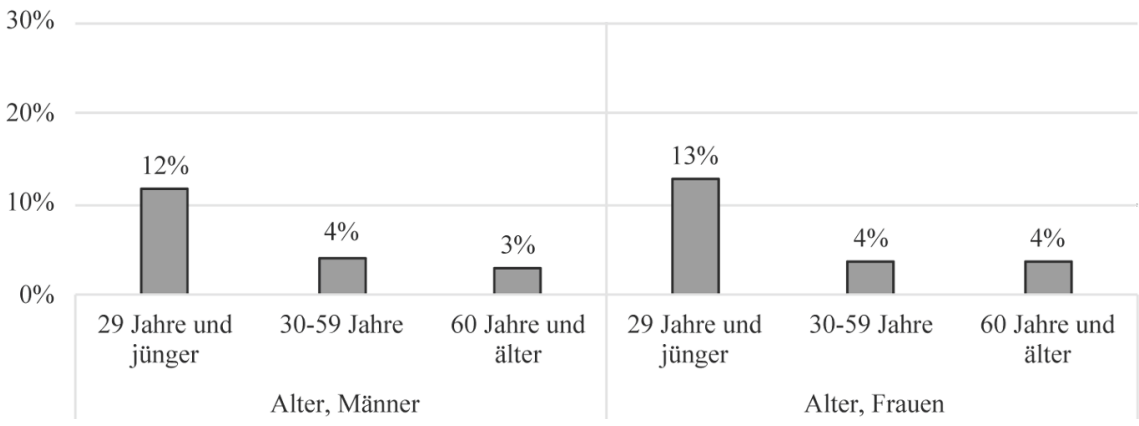

Auch hier ist, im Vergleich zur breiten Unterstützung und der intendierten Teilnahme oben, ein deutlicher Altersunterschied zu erkennen. Unter den Befragten nehmen häufiger junge Menschen im Alter von 16 bis 29 Jahren an den Fridays for Future-Protesten teil, wobei sich zwischen den Geschlechtern nur leichte Unterschiede zeigen.

Im Folgenden vergleichen wir die Ergebnisse mit den Protestbefragungen, die im März 2019 vom Institut für Protest- und Bewegungsforschung in Berlin und Bremen durchgeführt wurden (Abb. 10.8). Einschränkend muss jedoch hinzugefügt werden, dass die Teilnahme an der GIP-Befragung erst ab einem Mindestalter von 16 Jahren möglich ist und es somit zu Verzerrungen der Ergebnisse im Vergleich mit den Daten der ipb-Befragung, deren Mindestalter 14 Jahre beträgt, kommen kann.

Wenig überraschend finden auch wir, dass die Teilnehmer_innen sich überwiegend aus der jüngsten Altersgruppe rekrutieren. Die Gruppe der 15bis 25-Jährigen stellt in der GIP-Befragung 38 Prozent der Teilnehmer_innen, in der Protestbefragung des ipb sogar einen deutlich höheren Anteilvon 71 Prozent (Sommer u. a. 2019). Auch über die anderen Altersgruppen hinweg finden 
Abbildung 10.8: Vergleich der Protestteilnehmer_innen der beiden Befragungen ipb (März 2019) und GIP (Mai 2019)

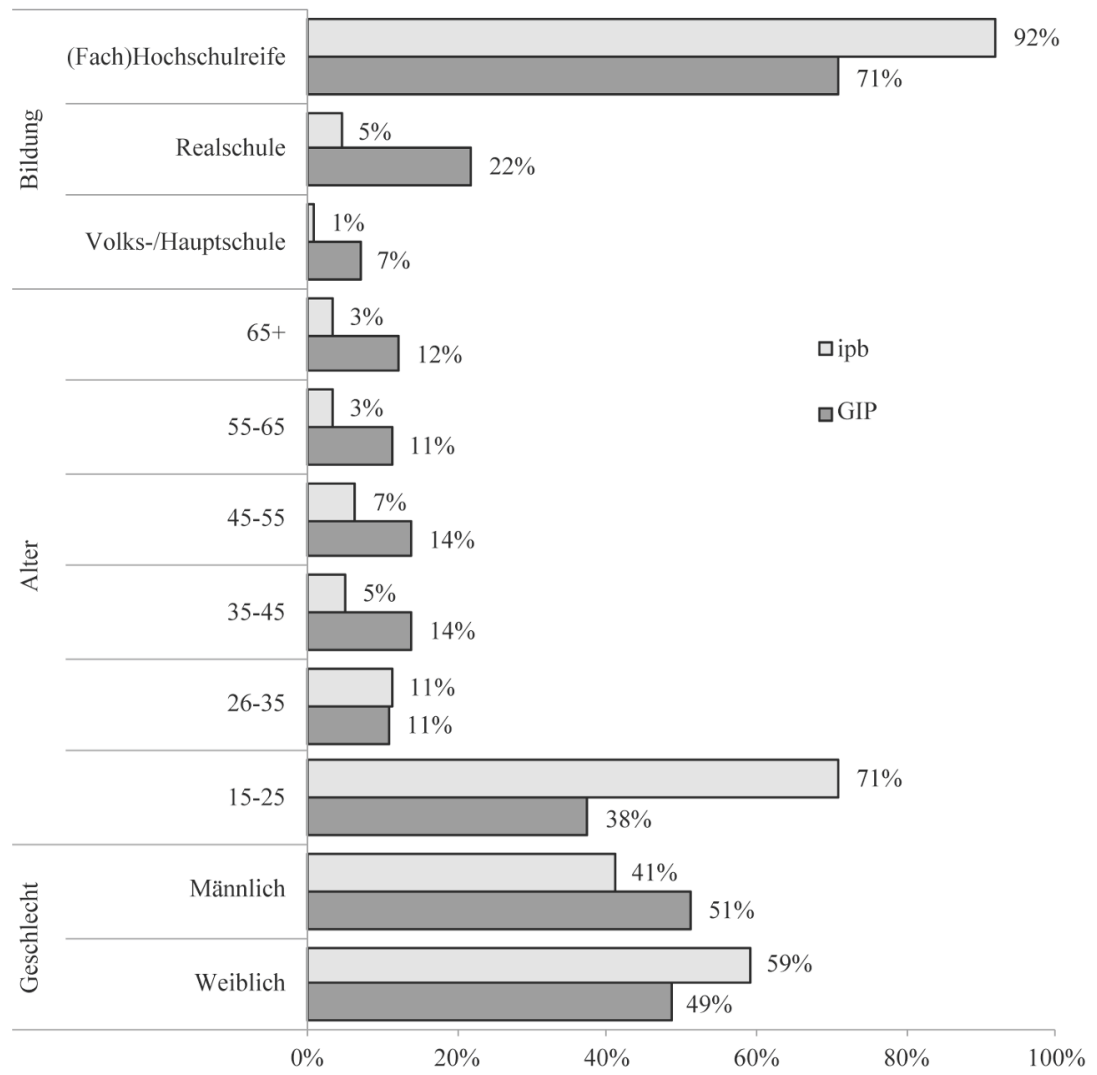

Hinweis: Für die Schüler_innen im GIP Survey war die Schulart nicht ausgewiesen, sodass wir diese aus Gründen der Vergleichbarkeit hier herausgenommen haben.

wir anteilsmäßig Diskrepanzen - mit Ausnahme der 26- bis 35-Jährigen, die in beiden Befragungen gleichauf sind (Sommer u. a. 2019). Die Unterschiede lassen sich durch die verschiedenen Zeitpunkte der beiden Befragungen erklären und deuten bereits auf den Beginn der Veränderung in der Zusammensetzung der Bewegung hin. Während die Bewegung im März 2019 beim 1. Globalen Klimastreik noch überwiegend von Schüler_innen geprägt war (vgl. auch Koos 2019), wurde sie im Verlauf der folgenden Monate zunehmend von Menschen höherer Alterssegmente unterstützt (Sommer 
u.a., Kapitel 2; Neuber u.a., Kapitel 3). Hinsichtlich der Altersstruktur hat sich die anfänglich reine Jugendbewegung innerhalb eines halben Jahres bis September $2019 \mathrm{zu}$ einer breiteren gesellschaftlichen Bewegung entwickelt (vgl. Koos/Lauth 2019). Im Mai, zum Zeitpunkt der GIP-Befragung, hat sich dieser Wandel bereits angedeutet.

Hinsichtlich der sozioökonomischen Zusammensetzung rekrutieren sich die Teilnehmer_innen mehrheitlich aus höheren Bildungsschichten, wie die Ergebnisse beider Befragungen zeigen. Die große Mehrheit (92 Prozent) der Befragten im März besitzt oder strebt eine (Fach-)Hochschulreife an (Sommer u. a. 2019). In der GIP-Befragung besitzt ebenfalls die Mehrheit (71 Prozent) einen solchen Abschluss. Die ipb-Protestbefragung im März konstatiert, dass mit 59 Prozent mehr Frauen als Männer an den Fridays for Future-Protesten teilnehmen (Sommer u. a. 2019). Die Daten des GIP lassen hingegen keine signifikanten Geschlechterunterschiede erkennen.

Die politische Orientierung ist nicht explizit grafisch im Vergleich dargestellt, wir finden dazu in beiden Befragungen Übereinstimmungen. Laut der ipb-Befragung identifizieren sich die meisten Protestteilnehmer_innen mit den Grünen und der Linken, also links der Mitte, wobei viele Teilnehmer_innen auch angeben, keine Parteipräferenz zu haben (Sommer u. a. 2019). In unseren Daten zeigt sich ebenfalls, dass sich unter Wähler_innen der Grünen und der Linken mit insgesamt 20 Prozent die meisten Protestteilnehmer_innen finden. Überdies ordnen sich in der ipb-Studie 72 Prozent der Befragten auf einer Skala von I (»links«) bis 10 (»rechts«) links der Mitte ein (Sommer u. a. 2019). In unseren Daten sind es mit 82 Prozent der Befragten links der Mitte sogar etwas mehr.

Hinsichtlich einer Mitgliedschaft in Umweltorganisationen stellt das ipb fest, dass unter den Schüler_innen vier Prozent und unter den Erwachsenen knapp ein Viertel (23,5 Prozent) passive Mitglieder sind. Knapp fünf Prozent der Schüler_innen sind aktiv in solchen Organisationen engagiert, unter den Erwachsenen sind es knapp neun Prozent (Sommer u. a. 2019). In den Daten der GIP-Befragung sehen wir ein geringeres Ausmaß an Engagement in Umweltorganisationen, wobei sich die Anteile aktiver und passiver Mitgliedschaft nicht wesentlich unterscheiden. Knapp zehn Prozent der Befragten geben an, passives Mitglied zu sein, während sich acht Prozent aktiv engagieren.

Welchen Einfluss hat nun die Unterstützung im Sinne einer positiven Meinung oder der aktiven Teilnahme an Protesten auf die Unterstützung politischer Forderungen und den Wandel des Lebensstils? 


\section{Fridays for Future, die Einführung einer CO2-Steuer und der Wandel der Lebensstile}

Unterstützt durch einige Wissenschaftler_innen hat Fridays for Future im Sommer einen Forderungskatalog aufgestellt. Eine der sechs Forderungen sieht die Einführung einer CO2-Steuer im Umfang von $180 €$ pro Tonne $\mathrm{CO}_{2}$ vor. Diese Forderung war von Anfang an sehr umstritten, da die Kosten bei einem durchschnittlichen Verbrauch von elf Tonnen $\mathrm{CO}_{2}$ pro Bürger_in von manchen als zu hoch angesehen wurden. In der Befragung des GIP wurde den Befragten der Vorschlag einer CO2-Steuer vorgelegt und erfragt, inwiefern eine solche Steuer unterstützt werden würde. ${ }^{2}$ Aus der Umfrageforschung weiß man, dass Befragte der Einführung von Steuern grundsätzlich kritisch gegenüberstehen. Der Steuerbegriff weckt bei vielen Befragten offenbar negative Assoziationen, sodass die Unterstützung für neue Steuern zumeist als relativ moderat angesehen werden kann, unabhängig davon, für welchen Zweck diese aufgewendet werden sollen. Wir finden ein entsprechend polarisiertes Bild, in welchem sich annähernd gleich viele Befragte für (35 Prozent) und gegen die CO2-Steuer aussprechen (39 Prozent), mit einem nicht unerheblichen Teil an Befragten, die sich nicht entscheiden können (26 Prozent). Die Unterstützung ist insbesondere bei jüngeren Befragten recht hoch (40 Prozent bei der jüngsten Gruppe). Männer lehnen eine solche Steuer eher $\mathrm{ab}$ als Frauen (siehe auch Koos/Nauman 2019). In welchem Zusammenhang steht nun die Unterstützung für Fridays for Future und die mögliche Einführung einer $\mathrm{CO} 2$-Steuer? In Abbildung 10.9 haben wir die Zustimmung zur Einführung einer solchen Steuer in Abhängigkeit der Einstellung zu Fridays for Future sowie der Teilnahme an Klimademonstrationen dargestellt.

2 »Die Schülerinnen und Schüler der `Fridays for Future«-Bewegung haben einen Forderungskatalog ausgearbeitet. Die Forderungen zielen auf die Einhaltung des Pariser Klimaschutzabkommens, welches die Erderwärmung auf 1,5 Crad Celsius begrenzen will. Unter anderem wird gefordert, eine Steuer von $180 €$ pro Tonne CO2-Emission einzuführen. Dies würde viele Lebensmittel und Alltagsgüter, aber vor allem Reisen und Autofahren deutlich teurer machen. Lehnen Sie die Einführung einer $\mathrm{CO}_{2}$-Steuer ab oder stimmen Sie dieser zu? « Antwortkategorien: »Stimme ich voll zu«, »stimme ich ein bisschen zu«und »lehne ich $a b \ll, » l e h n e$ ich stark $a b \ll$ wurden zusammengefasst. 
Abbildung 10.9: Zustimmung zu einer CO2-Steuer nach Art der Unterstützung für Fridays for Future

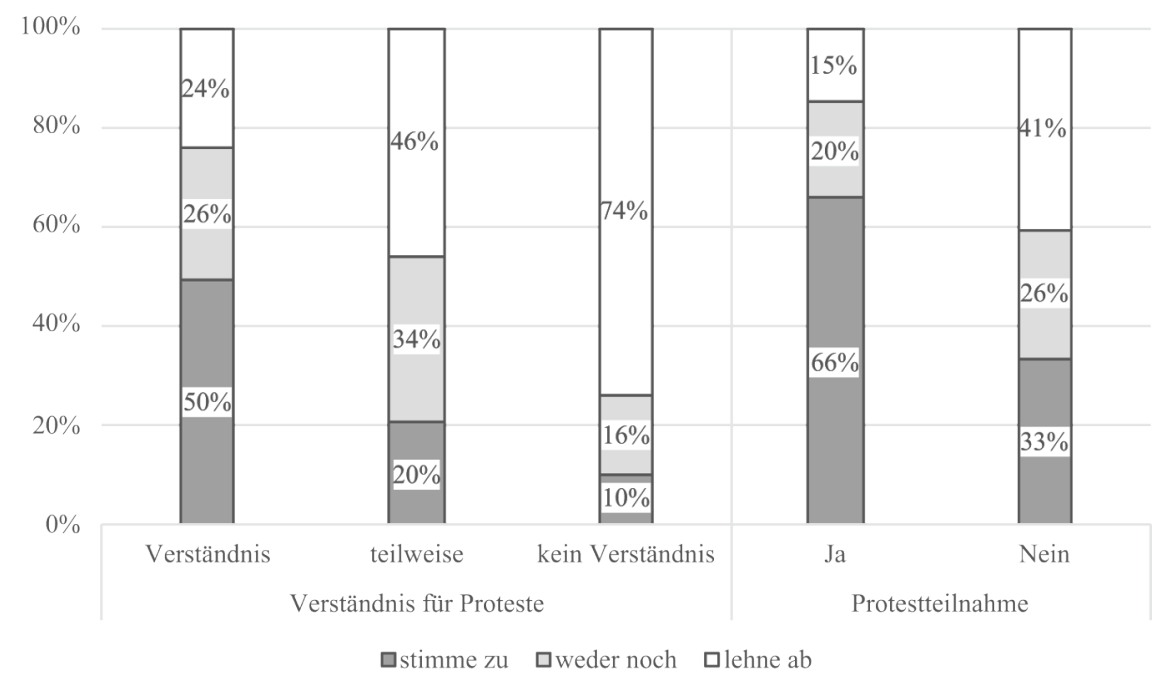

Es zeigt sich, dass die Unterstützer_innen von Fridays for Future auch einer Klimasteuer aufgeschlossen gegenüberstehen, jedoch nicht vollkommen geschlossen. Etwa die Hälfte der Befragten, die volles Verständnis für die Bewegung äußert, unterstützt die CO2-Steuer. Mit sinkendem Verständnis nimmt auch die Unterstützung für die Klimasteuer ab. Bei Teilnehmer_innen an Klimastreiks ist die Unterstützung zwar mit 60 Prozent sehr hoch, jedoch sind auch hier knapp 15 Prozent gegen eine solche Steuer. Bei den Nichtteilnehmer_innen zeigt sich ein differenzierteres Bild. Eine Mehrheit von 41 Prozent dieser Gruppe lehnt eine CO2-Steuer ab, knapp über ein Drittel (33 Prozent) befürwortet sie. Zusammenfassend stellen wir fest, dass die von Fridays for Future vorgeschlagene CO2-Steuer in Höhe von $180 €$ pro Tonne $\mathrm{CO}_{2}$ auch unter ihren Unterstützer_innen und den aktiven Teilnehmer_innen durchaus kontrovers gesehen wird. Dies kann viele Ursachen haben und darf keinesfalls als generelle Ablehnung klimapolitischer Maßnahmen verstanden werden. Eine Erklärung für die differenzierte Unterstützung könnte sein, dass, je nach Ausgestaltung der Steuer, Befürchtungen bestehen, diese könnte zu einer wachsenden sozialen Ungleichheit beitragen. In einem Befragungsexperiment unter Teilnehmer_innen einer Fridays for Future-Demonstration 
finden wir heraus, dass die Zustimmung zu einer $\mathrm{CO} 2$-Steuer sinkt, wenn durch die Steuer die soziale Ungleichheit ansteigen würde (Koos/Lauth 2019). Die Vorstellungen darüber, mittels welcher politischen Maßnahmen Klimaschutz am besten umgesetzt werden kann und wie sowohl Klimaschutz als auch Sozialverträglichkeit vereint werden können, unterscheiden sich wenig überraschend auch innerhalb der Fridays for Future-Bewegung.

Eine weitere Forderung von Fridays for Future richtet sich auf den Wandel der privaten Lebensführung in Richtung eines klimaneutralen Lebensstils. Im GIP wurde deshalb auch nach einer Änderung des eigenen Konsumverhaltens gefragt. ${ }^{3}$ Die Ergebnisse sind in Abbildung 10.10 dargestellt.

Es zeigt sich, dass zwei Drittel der Unterstützer_innen ihren Lebensstil seit Anfang 2019 etwas oder sogar stark verändert haben und etwa auf Flugreisen verzichten oder weniger Fleisch konsumieren. Unter denjenigen, die kein Verständnis gegenüber der Klimabewegung zeigen, hat hingegen nur knapp über ein Drittel seinen Konsumstil verändert. Bei den aktiven Protestteilnehmer_innen verzeichnen wir die stärkste Veränderung hinsichtlich privater Konsumgewohnheiten. So gibt knapp die Hälfte dieser Gruppe an, ihren Lebensstil stark verändert zu haben, weitere 41 Prozent haben ihn etwas verändert. Auffallend ist auch, dass unter denjenigen, die nicht am Protest teilnehmen, immerhin über die Hälfte der Befragten ihren Lebensstil zumindest etwas verändert haben. Dies deutet darauf hin, dass die Klimabewegung auch außerhalb ihres aktiven Kerns einen nicht unbedeutenden Einfluss auf die private Lebensführung von Bürger_innen ausübt.

3 »Um dem Klimawandel entgegenzuwirken, fordern die Jugendlichen auch, den eigenen Lebensstil zu verändern. Das heißt beispielsweise, auf Flugreisen zu verzichten oder weniger Fleisch zu essen. Haben Sie seit Anfang 2019 Ihren Lebensstil verändert, um das Klima zu schützen?« 
Abbildung 10.10: Änderungen des eigenen Lebensstils nach Art der Unterstützung für Fridays for Future

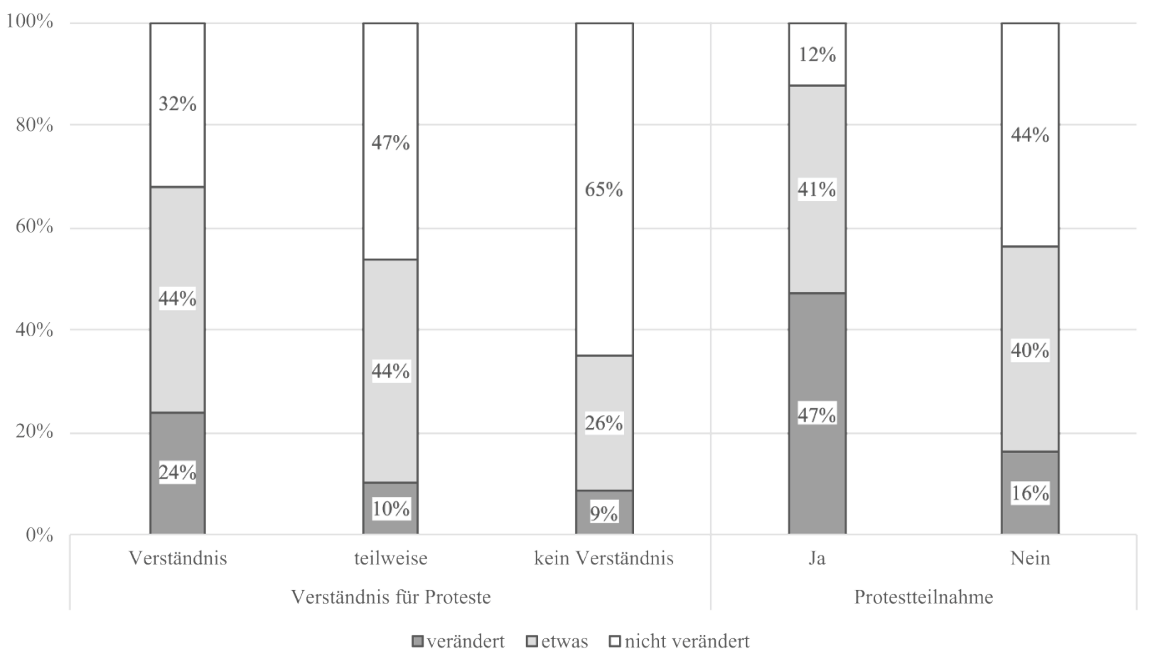

Hinweis: Die Antwortkategorien »sehr viel« und »viel« wurden zu »verändert« zusammengefasst; »ein wenig« und »überhaupt nicht« wurden zu »nicht verändert« zusammengefasst.

\section{Zusammenfassung und Ausblick}

Insgesamt belegen die Ergebnisse unserer Umfrage eine große Unterstützung für Fridays for Future, die jedoch sozialstrukturell variiert. Für alle drei Formen der Unterstützung zeigt sich, dass die Klimabewegung insbesondere durch Menschen jüngeren Alters, Menschen mit hohem Bildungsstand und Wähler_innen linker Parteien die größte Unterstützung erfährt. Wie auch andere Studien finden wir ausgeprägte Geschlechterunterschiede. Vor allem junge Frauen bringen ihre Unterstützung für Fridays for Future, sowohl durch eine stärker positive Einstellung gegenüber der Bewegung als auch durch eine höhere intendierte Protestteilnahme, zum Ausdruck. Dies führt, wie die Daten der Protestbefragungen im Kapitel 2 zeigen, auch zu einer größeren aktiven Teilnahme von Frauen an den Klimaprotesten. Insgesamt lassen sich für Fridays for Future ähnliche sozialstrukturelle Gradienten identifizieren, wie sie in bereits vorliegenden Arbeiten zu Ein- 
stellungen hinsichtlich sozialer Bewegungen und Umweltschutz diskutiert wurden.

Der Vergleich der Daten mit den Protestbefragungen in Bremen und Berlin vom März 2019 zeigt, dass die Ergebnisse hinsichtlich der Alters- und Bildungsstruktur als auch der politischen Orientierung der Protestteilnehmer_innen große Ähnlichkeiten aufweisen. Durch den Vergleich wird auch deutlich, dass sich die Zusammensetzung der Teilnehmer_innen - der innere konzentrische Kreis und Kern der Bewegung - gewandelt hat und sich bereits im Mai 2019, nur zwei Monate nach dem 1. Globalen Klimastreik am 15. März, deutlich heterogener ist. Zukünftige Forschung sollte untersuchen, inwiefern die geplante Protestteilnahme wirklich $\mathrm{zu}$ einer tatsächlichen Teilnahme führt und welche Faktoren einer solchen im Wege stehen. Dies ist insbesondere von Relevanz, da die vorliegenden Daten zeigen, wie groß der Anteil derer ist, die sich vorstellen können, sicher oder wahrscheinlich an zukünftigen Protesten teilzunehmen und daher ein großes Potenzial an Ressourcen für die junge Klimabewegung darstellen.

Die Unterstützung der Bewegung äußert sich nicht zuletzt auch in der Bereitschaft, neue klimapolitische Maßnahmen wie etwa eine CO2-Steuer mitzutragen, auch wenn diese mit hohen Kosten verbunden sind. Wir finden, dass mit zunehmendem Unterstützungsgrad der Klimabewegung auch die Zustimmung zu einer CO2-Steuer zunimmt. Eine einstimmige Befürwortung einer solchen politischen Maßnahme findet sich allerdings selbst unter den Protestteilnehmer_innen nicht. Daher sollte in zukünftiger Forschung weiter untersucht werden, welche Faktoren die Unterstützung einer CO2Steuer bedingen und wie sich die Heterogenität innerhalb der Fridays for Future-Unterstützer_innen erklären lässt. Zuletzt hat die Unterstützung der Bewegung auch Einfluss auf den Wandel der privaten Lebensführung hin zu einem klimaneutralen Lebensstil. Wie weitreichend und langfristig die berichteten Verhaltensänderungen sind, lässt sich aber bislang nicht beurteilen.

\section{Literatur}

Andrews, Kenneth T./Beyerlein, Kraig/Farnum, Tucker T. (2015): The Legitimacy of Protest: Explaining White Southerners' Attitudes Toward the Civil Rights Movement, in: Social Forces 94, H. 3, S. 1021-1044. 
Blocker, T. Jean/Eckberg, Douglas Lee (1997): Gender and Environmentalism: Results from the 1993 General Social Survey, in: Social Science Quarterly 78, H. 4, S. 841-858.

Dietz, Thomas/Kalof, Linda/Stern, Paul C. (2002): Gender, Values, and Environmentalism, in: Social Science Quarterly 83, H. 1, S. 353-364.

Dietz, Thomas/Stern, Paul C./Guagnano, Gregory A. (1998): Social Structural and Social Psychological Bases of Environmental Concern, in: Environment and Behaviour 30, S. 450-471.

Ennis, James G./Schreuer, Richard (1987): Mobilizing Weak Support for Social Movements: The Role of Grievance, Efficacy, and Cost, in: Social Forces 66, H. 2, S. 390-409.

Franzen, Axel/Meyer, Reto (2010): Environmental Attitudes in Cross-National Perspective: A Multilevel Analysis of the ISSP 1993 and 2000, in: European Sociological Review 26, H. 2, S. 219-234.

Jones, Robert Emmet/Dunlap, Riley E. (1992): The Social Bases of Environmental Concern: Have They Changed over Time?, in: Rural Sociology 57, H. 1, S. 28-47.

Klandermans, Bert (1984), Mobilization and Participation: Social-Psychological Expansisons of Resource Mobilization Theory, in: American Sociological Review 49, H. 5, S. 583-600.

Klandermans, Bert/Oegema, Dirk (1987): Potentials, Networks, Motivations, and Barriers: Steps towards Participation in Social Movements, in: American Sociological Review 52, H. 4, S. 519-531.

Koos, Sebastian (2019): Klima-Aktivismus von jungen Menschen. Ergebnisse einer Befragung unter den Teilnehmenden am »Fridays for Future«Schulstreik in Konstanz, 15. März 2019.

Koos, Sebastian/Lauth, Franziska (2019): Die Entwicklung und Zukunft der Fridays for Future-Bewegung. Ergebnisse von zwei Befragungen während der Fridays for Future-Demonstrationen in Konstanz am 24. Mai und 20. September 2019, Forschungsbericht, http://nbn-resolving.de/ urn:nbn:de:bsz:352-2-1vspckwsvnogm8.

Koos, Sebastian/Naumann, Elias (2019): Vom Klimastreik zur Klimapolitik. Die gesellschaftliche Unterstützung der »Fridays for Future«-Bewegung und ihrer Ziele. Forschungsbericht, http://nbn-resolving.de/ urn:nbn:de:bsz:352-2-1jdetkrk6b9yl4. 
Kvaløy, Berit/Finseraas, Henning/Listhaug, Ola (2012): The Publics' Concern for Global Warming: A Cross-national Study of 47 Countries, in: Journal of Peace Research 49, H. 1, S. 11-22.

McCarthy, John D./Zald, Mayer N. (1977): Resource Mobilization and Social Movements: A Partial Theory, in: American Journal of Sociology 82, H. 6, S. 1212-1241.

McCright, Aaron M./Dunlap, Riley E./Marquart-Pyatt, Sandra T. (2016): Political Ideology and Views about Climate Change in the European Union, in: Environmental Politics 25, H. 2, S. 338-358.

Muñoz, Jordi/Anduiza, Eva (2019), »If a Fight Starts, watch the Crowd«: The Effect of Violence on Popular Support for Social Movements, in: Journal of Peace Research 56, H. 4, S. 485-498.

Neumayer, Eric (2004): The Environment, Left-wing Political Orientation and Ecological Economics, in: Ecological Economics 51, H. 3, S. 167-175.

Olsen, Marvin E. (2014), Perceived Legitimacy of Social Protest Actions, Social Problems, 15, H. 3, S. 297-310.

Rohrschneider, Robert (1990): The Roots of Public Opinion toward New Social Movements: An Empirical Test of Competing Explanations, in: American Journal of Political Science 34, H. 1, S. 1-30.

Sommer, Moritz/Rucht, Dieter/Haunss, Sebastian/Zajak, Sabrina (2019): Fridays for Future. Profil, Entstehung und Perspektiven der Protestbewegung in Deutschland, ipb working paper 2.2019, Berlin: Institut für Protest- und Bewegungsforschung, https://protestinstitut.eu/ipb-wor king-papers.

Stern, Paul C./Dietz, Thomas/Abel, Troy/Guagnano, Gregory A./Kalof, Linda (1999): A Value-Belief-Norm Theory of Support for Social Movements: The Case of Environmentalism, in: Human Ecology Review 6, H. 2, S. 81-97.

Thomas, Emma F./Louis, Winnifred R. (2013): When Will Collective Action Be Effective? Violent and Non-Violent Protests Differentially Influence Perceptions of Legitimacy and Efficacy Among Sympathizers, in: Personality and Social Psychology Bulletin 40, H. 2, S. 263-276.

Tjernström, Emilia/Tietenberg, Tom (2008): Do Differences in Attitudes Explain Differences in National Climate Change Policies?, in: Ecological Economics 65, H. 2, S. 315-324. 
Van Liere, Kent D./Dunlap, Riley E. (1980): The Social Bases of Environmental Concern: A Review of Hypotheses, Explanations and Empirical Evidence, in: The Public Opinion Quarterly 44, H. 2, S. 181-197.

Van Stekelenburg, Jacquelien/Klandermans, Bert (2013): The Social Psychology of Protest, in: Current Sociology 61, H. 5/6, S. 886-905. 